\title{
Re-emergent tremor of Parkinson's disease
}

\author{
Joseph Jankovic, Kenneth S Schwartz, William Ondo
}

\begin{abstract}
Objective-To characterise postural tremors in patients with Parkinson's disease. Rest tremor is a well recognised cardinal symptom of Parkinson's disease, but postural tremors associated with the disease may cause more disability than the more typical rest tremor. Postural tremor of Parkinson's disease has been attributed to enhanced physiological tremor, clonus, or coexistent essential tremor. It is postulated that one type of postural tremor in Parkinson's disease represents a rest tremor that re-emerges after a variable delay while maintaining posture, hence "re-emergent tremor".

Methods-Accelerometry, peak frequency, peak frequency amplitude, root mean square (RMS) amplitude, and latency were determined in 18 patients (mean age: 63.2 (SD 9.8) years) with Parkinson's disease who had clinically evident postural tremor, 20 (mean age: 66.9 (SD 5.8) years) with typical essential tremor, and seven (mean age: 68.7 (SD 15.3) years) with the combination of pre-existing essential tremor and subsequent Parkinson's disease (essential tremor/Parkinson's disease). Latency, the time interval starting with the assumption of an outstretched posture and ending with the onset of postural tremor, was measured by marking the start time by a pulse produced from interrupting a beam to a photocell when the arm reached a horizontal position.
\end{abstract}

Results-The latency for the re-emergent tremor (9.37 (SD 10.66) s), present in 12 of 18 patients with Parkinson's disease, was significantly $(p<0.0005)$ longer than the latency for postural tremor of essential tremor $(1.29 \mathrm{~s}$ in one patient, absent in 19 others); five of seven essential tremorl Parkinson's disease patients had an observed latency (6.57 (SD $8.23 \mathrm{~s}$ ) which was also significantly $(p<0.005)$ longer than that for essential tremor. There was no difference in the mean tremor frequency $(\sim 5.5 \mathrm{~Hz})$ between the re-emergent tremor and the more typical Parkinson's disease rest tremor. The amplitudes were generally higher for the postural tremor associated with Parkinson's disease compared with those of essential tremor.

Conclusion-These studies suggest that the re-emergent tremor of Parkinson's disease can be differentiated from the postural tremor of essential tremor and that it may share pathophysiological mechanisms with the more typical rest tremor.

(F Neurol Neurosurg Psychiatry 1999;67:646-650)

Keywords: tremor; essential tremor; Parkinson's disease; re-emergent tremor

In his original Essay on the Shaking Palsy James Parkinson ${ }^{1}$ emphasised rest tremor as the characteristic tremor of Parkinson's disease. He described the tremor as "Involuntary tremulous motion, with lessened voluntary muscular power, in parts, not in action, and even supported ... that agitation continues in full force whilst the limb is at rest and unemployed; and even is sometimes diminished by calling the muscles into employment." Parkinson ${ }^{1}$ and later Charcot $^{2}$ clearly differentiated between the typical rest tremor of Parkinson's disease and the postural tremor of essential tremor. Gowers, ${ }^{3}$ however, noted that the two types of tremor may overlap and that "some cases are met with a character intermediate between the two affections". Gelma ${ }^{4}$ also recognised an "intermediate" form of tremor which he considered to represent an overlap between Parkinson's disease and essential tremor.

Rest tremor is a well recognised cardinal feature of Parkinson's disease, but many patients have a postural tremor that is more prominent and disabling than the classic rest tremor. Postural tremor without parkinsonian features and without any other known aetiology is often diagnosed as essential tremor, but isolated postural tremor may be the initial presentation of Parkinson's disease, and it may be found with higher than expected frequency in relatives of patients with Parkinson's disease. ${ }^{5}$ Although some authors have claimed that "clinically and electrophysiologically, the postural tremors of essential tremor and Parkinson's disease are indistinguishable", 6 we present evidence that the two forms of postural tremor can be differentiated both clinically and physiologically.

We have found that the postural tremor in patients with essential tremor usually occurs as soon as the limb assumes a horizontal posture, whereas in some patients with Parkinson's disease, the postural tremor emerges after a variable latency during which time no observable postural tremor is present. It is this latency 
that, we think, differentiates the postural tremor associated with Parkinson's disease from the postural tremor of essential tremor. The primary objective of this study was to characterise this re-emergent tremor of Parkinson's disease and to determine which clinical and physiological variables differentiate this tremor from the typical rest tremor of Parkin- son's disease and the postural tremor of essential tremor.

\section{Methods}

Consecutive patients with Parkinson's disease, essential tremor, or both, who had obvious postural tremor were recruited from the Parkinson's Disease Center and Movement
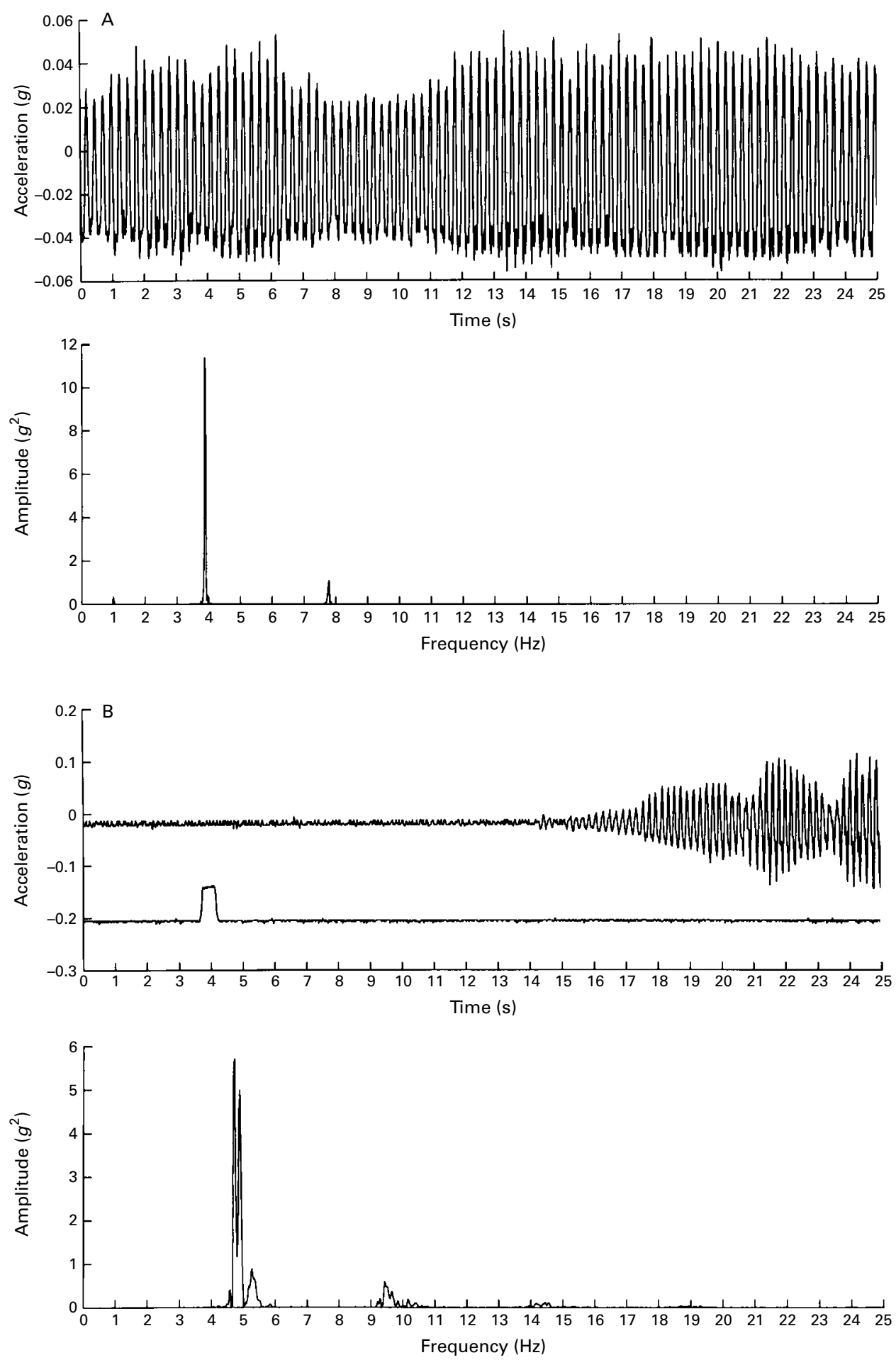

(A) A recording of a continuous rest tremor in a patient with Parkinson's disease, peak frequency of $4.5 \mathrm{~Hz}$. (B) $\mathrm{A}$ recording of a re-emergent tremor in the same patient after a latency of 12 seconds from the start of the horizontal position, marked by an artifact produced by the interruption of a light beam pointed at a photocell detector. The frequency of this tremor is similar to that of the rest tremor. 
Disorders Clinic, Baylor College of Medicine. The postural tremor was evident during maintenance of an antigravity posture. The diagnosis of Parkinson's disease was based on the finding of at least three of the four cardinal signs: rest tremor, bradykinesia, rigidity, and postural instability. Patients with essential tremor met the diagnostic criteria for "definite" essential tremor formulated by the Tremor Research and Investigation Group $(\text { TRIG })^{7}$ : bilateral postural tremor involving hands or forearms, which was visible and persistent, lasting longer than 5 years, and without any other apparent cause of tremor. For the purposes of this study, re-emergent tremor is defined as a tremor which occurs after a finite period (latency) from the time the subject assumes horizontal posture of the arm to the onset of the wrist and/or finger tremor.

The patient is comfortably seated in a chair and the upper limb most severely affected by postural tremor, as determined by clinical observation and patient history, is selected for the recording. A piezoresistive accelerometer, with a band width from $0-250 \mathrm{~Hz}$, weighing about $5 \mathrm{~g}$ is attached to the dorsal surface of the hand above the distal head of the third metacarpal with a velcro band firmly encircling the hand at the level of the distal metacarpal heads of digits two through five. The axis of sensitivity of the accelerometer is oriented so that it is perpendicular to the dorsal surface of the hand. The signal is amplified, AC coupling its output to remove a DC shift proportional to the accelerometer's position relative to the earth's gravitational axis. Amplified outputs are linearly scaled so that a $5 \mathrm{~V}$ output represents a $1 \mathrm{~g}$ acceleration. The amplified accelerometer signal is recorded using a Tektronix THS710 digital storage oscilloscope. Each sample is digitised as a 2500 point, 8 bit waveform per channel. The data, scaled for oscilloscope gain coefficients, is transferred to a windows NT computer using a serial RS232 interface. The peak frequency was computed using power spectral density (PSD) calculations, reporting the peak frequency as well as the amplitude at this peak frequency $\left(\mathrm{g}^{2}\right)$. Additionally, root mean square (RMS) amplitude, the mean value of the square root of the square value which is equivalent of the mean value of the waveform after rectification, of the 50 second epoch is computed.

The upper limb tremor is recorded in three positions, each held for a 50 second interval: (1) at rest: forearm supported on an chair arm rest and the hand unsupported; (2) postural: arm held in an outstretched, horizontal, prone

Physiological characteristics of postural tremors

\begin{tabular}{lcccl}
\hline Variable & $E T^{4}(n=20)$ & $E T / P D^{B}(n=7)$ & $P D^{C}(n=18)$ & $p$ Value \\
\hline Latency $(\mathrm{s})$ & $0.06(0.28)$ & $4.70(7.57)$ & $6.25(9.76)$ & $\mathrm{AB}<0.01$ \\
& $(0-1.29)$ & $(0-23.0)$ & $(0-47.0)$ & $\mathrm{AC}<0.01$ \\
Frequency $(\mathrm{Hz})$ & $5.50(0.88)$ & $5.75(1.23)$ & $5.47(1.24)$ & $\mathrm{NS}$ \\
Acceleration $\left(\mathrm{g}^{2}\right)$ at & $(3.5-7.2)$ & $(4.3-7.7)$ & $(4.1-9.6)$ & \\
peak frequency & $(0.002-4.07)$ & $11.20(10.70)$ & $3.83(6.82)$ & $\mathrm{AB}<0.0001$ \\
RMS (g) & $0.051(0.053)$ & $0.02-28.74)$ & $(0.004-26.93)$ & $\mathrm{AC}<0.05$ \\
& $(0.013-0.217)$ & $(0.07-0.75)$ & $0.186(0.115)$ & $\mathrm{AC}<0.0001$ \\
& & $(0.013-0.370)$ & $\mathrm{AB}<0.0005$ \\
\hline
\end{tabular}

Values represent means (SD) (range); AB refers to a comparison between ET and ET/PD, AC refers to a comparison between ET and PD. position; (3) wing beating: arm held in a horizontal position the palms down, shoulders abducted, and elbow flexed, about 1 inch in front of the tip of the nose. To measure the latency, after resting arms on their thighs in a seated position for at least 1 minute, the patients are instructed to raise their arms up to the outstretched position and to maintain the posture for at least 50 seconds. In the process of assuming this new position the hand momentarily interrupts a light beam which is pointed at a photocell detector that is positioned at the patient's shoulder level. The photocell output is simultaneously recorded as a second channel. The instant (the leading edge of the pulse) the patient breaks the beam (at the horizontal position), marked by a pulse artifact, indicates the start of the newly achieved horizontal position and is considered the start of the latency time interval. The latency of onset of tremor is measured as the time interval between the leading edge of the pulse and the onset of tremor exceeding the amplitude of the resting limb activity.

Comparisons between the three populations were performed using $F$ test and paired $t$ test as well as Pearson's $\chi^{2}$ analyses (where indicated). Significance levels of comparisons are indicated as $\mathrm{p}<0.05$.

\section{Results}

There were 45 patients categorised as either essential tremor $(n=20)$, Parkinson's disease $(n=18)$, or essential tremor/Parkinson's disease $(n=7)$. Their ages were similar (mean 66.9, 63.2, and 68.7 years, respectively), but the patients with essential tremor had symptoms on the average 15 years longer than those with Parkinson's disease. By contrast with patients with essential tremor, who had no observable latency, except for one patient whose latency was 1.29 seconds, five of seven patients with essential tremor/Parkinson's disease $(\mathrm{p}<0.005)$ and 12 of 18 patients with Parkinson's disease $(\mathrm{p}<0.0005)$ had re-emergent tremor after a well defined latency (figure) (table). When patients without re-emergent tremor were excluded, the mean latency (SD) for patients with essential tremor/Parkinson's disease was 6.57 (8.23) seconds (range: 1.35-23.0), and for those with Parkinson's disease alone the mean latency increased to 9.37 (10.66) seconds (range: 1.0-47.0). The mean frequency (SD) of rest tremor in patients with Parkinson's disease (4.91 (1.32) Hz, range: 3.76-9.1) was similar to that of re-emergent tremor (5.47 (1.24) $\mathrm{Hz}$, range: 4.1-9.6). Except for the expected absence of rest tremor in patients with essential tremor, there was no difference in the amplitude of the rest and wing beating tremors in the three groups of patients as measured by acceleration at peak frequency $\left(\mathrm{g}^{2}\right)$ and RMS (g). Postural tremor with arms outstretched, however, had a higher amplitude in patients with essential tremor/Parkinson's disease and in Parkinson's disease compared with essential tremor alone (table). There was no correlation between severity of parkinsonian findings, as measured by the unified Parkin- 
son's disease rating scale (UPDRS), ${ }^{8}$ and the occurrence of re-emergent tremor.

\section{Discussion}

In this study of 18 patients with Parkinson's disease, 20 with essential tremor, and seven with essential tremor/Parkinson's disease we found that $12(67 \%)$ patients with Parkinson's disease and only $5 \%$ of those with essential tremor had postural tremor that occurred after a significant delay. The mean latency of $10 \mathrm{sec}-$ onds (up to 47 seconds) for the re-emergent tremor was significantly longer than that for the postural tremor of essential tremor $(p<0.0005)$; the latency for the re-emergent tremor in patients with essential tremor/ Parkinson's disease was intermediate. The mean tremor frequency, $5.5 \mathrm{~Hz}$, was similar for the rest and postural tremors of Parkinson's disease suggesting that the two types of tremors were generated by the same central oscillator.

Tremors have been divided traditionally into rest and action (postural and kinetic) tremors. Rest tremor, one of the cardinal features of Parkinson's disease, is present sometime during the course of the disease in $68 \%-100 \%$ of pathologically proved cases of the disease..$^{9} 10$ Some patients, however, exhibit isolated postural tremor as the initial or predominant manifestation of Parkinson's disease. The mechanism of this postural tremor is unknown, but striatal ${ }^{18} \mathrm{~F}$-dopa uptake, measured by PET, is decreased in some patients with this isolated postural tremor, thus suggesting that it may be related to nigrostriatal dopamine deficiency. ${ }^{6}{ }^{11}$ Nigrostriatal dopamine deficiency has been postulated to underlie the typical Parkinson's disease rest tremor, but there is little or no correlation between rest tremor and striatal ${ }^{18} \mathrm{~F}$-dopa uptake in patients with Parkinson's disease. ${ }^{12}$

Because rest tremor is considered the typical parkinsonian tremor, most studies of tremor in Parkinson's disease have focused primarily on this tremor type and relatively little attention has been paid to the parkinsonian action tremor. It is the action tremor, however, that is often more troublesome for the patient. By contrast with rest tremor, which may produce a social handicap, the action tremors associated with Parkinson's disease correlate directly with motor disability ${ }^{13}$ and contribute to weakness and bradykinesia. ${ }^{14}$ Although sometimes referred to as "atypical" tremor, ${ }^{15}$ we think that re-emergent tremor is a characteristic, but often unrecognised, parkinsonian tremor.

The mechanisms of postural tremors in Parkinson's disease are not well understood and previous physiological studies have provided conflicting findings. Lance et $a l^{16}$ reported a 7-12 Hz "action tremor" during muscle contraction in 23 of 40 patients with Parkinson's disease, some of whom did not have rest tremor. They differentiated this "action" tremor from rest tremor on the basis of frequency and mode of onset (muscle relaxation $v$ muscle contraction). They also noted a minority of patients with "marked resting tremor which persisted during contraction with little change in frequency". They concluded that postural tremor in most patients with Parkinson's disease was a type of enhanced physiological tremor seen as a manifestation of the cogwheel phenomena. Teräväinen and Calne ${ }^{17}$ expanded on this finding when they recorded an "action tremor" of between 6 and $9 \mathrm{~Hz}$ in 26 of 36 patients with Parkinson's disease triggered by voluntary contraction of the biceps muscle. They concluded that the action tremor in these patients resulted from oscillation in an internal feedback circuit triggered by voluntary movement and that "resting and action tremor in parkinsonism share similar pathophysiologic properties". Others have argued that the postural tremor of Parkinson's disease was related to clonus and rigidity. ${ }^{18} 19$ The finding that one type of postural tremor in Parkinson's disease occurs after a delay of a few seconds suggests that, by contrast with essential tremor, this form of tremor, although centrally driven, is influenced by peripheral feedback. Microelectrode recordings have identified cells firing at rest tremor frequency in the contralateral ventrolateral thalamus ${ }^{20}$ and the globus pallidus internus. ${ }^{21}$ Neuronal discharges in these structures are markedly influenced by sensory input. It is, therefore, possible that sensory feedback generated by repositioning of the limb from rest to the outstretched posture or into the wing beating position briefly and transiently desynchronises the thalamic or pallidal discharges, which accounts for the delay in the appearance of the postural tremor. The critical role of these structures in the generation of rest tremor and postural tremors is supported by the finding that both types of tremor are improved by pallidotomy ${ }^{21}$ and by high frequency stimulation of the thalamus. ${ }^{22}$

In this study we provide evidence that patients with Parkinson's disease have at least two types of tremor present during a maintenance of posture: with and without latency. We postulate that the postural tremor without latency, seen in a third of our patients with Parkinson's disease, represents either enhanced physiological tremor ${ }^{16}$ or coexistent essential tremor, ${ }^{5324}$ whereas the Parkinson's disease postural tremor occurring after a latency, seen in the other two thirds of our patients with Parkinson's disease, represents a re-emergent rest tremor. The second hypothesis is supported by the finding that the re-emergent tremor has a frequency similar to that of Parkinson's disease rest tremor. Furthermore, all our patients in whom levodopa improved rest tremor reported that levodopa also improved their re-emergent postural tremor. As the primary aim of this study was to characterise physiologically reemergent tremor, we did not match the groups for amplitude (severity) of tremor and we did not objectively assess the response of this tremor to dopaminergic therapy. The response of postural tremors to pharmacological manipulation, however, should be explored in future studies. In this regard, it is noteworthy to point out that the UPDRS refers only to "tremor at rest" and "action or postural tremor of hands", but it does not specify which "action" tremor should be rated. As the latency is not often found without accurate measure- 
ments, many clinicians consider the reemergent tremor as postural tremor. Future studies should seek to determine the incidence and prevalence of re-emergent tremor and attempt to identify demographic, clinical, physiological, and pharmacological variables that predict the occurrence and severity of this type of tremor.

We thank the National Parkinson Foundation, the RSMIS foundation, and the TLL Temple Foundation for their generous support.

1 Parkinson J. An essay on the shaking palsy. London: Sherwood, Neely, and Jones, 1817:1-66.

2 Charcot JM. Lectures on the diseases of the nervous system. London: The New Sydenham Society, 1877:130-40.

3 Gowers WR. Manual of diseases of the nervous system. London: Churchill Livingston, 1888;2:636-59.

4 Gelma E. Les tremblements séniles Parkinsoniens et le tremblement rhythme oscillatoire de Demange. Rev Méd Est 1913;14:33-6.

5 Jankovic J, Beach J, Schwartz K, et al. Tremor and longevity in relatives of patients with Parkinson's disease, essential in relatives of patients with Parkinson's disease, essenta

tremor and control subjects. Neurology $1995 ; 45: 645-8$.
6 Brooks D, Playford ED, Ibanez V, et al. Isolated tremor and disruption of the nigrostriatal dopaminergic system: an ${ }^{18}$ F-dopa PET study. Neurology 1992;42:1554-60.

7 Findley LJ, Koller WC. Definitions and behavioral classifiFindley LJ, Koller WC. Definitions and behavioral classifi-
cations. In: Findley LJ, Koller W. Handbook of tremor disorcations. In: Findley LJ, Koller W. Handbook
ders. New York: Marcel Dekker, 1995:1-5.

8 Fahn S, Elton RL, members of the UPDRS Development Committee. Unified Parkinson's disease rating scale. In Fahn S, Marsden CD, Calne DB, et al, eds. Recent developments in Parkinson's disease. Vol 2. Florham Park, NJ: Macmillan Health Care Information, 1987:153-64.

9 Rajput AH, Rozdilsky B, Ang L. Occurrence of resting tremor in Parkinson's disease. Neurology 1991;41:1298-9.

10 Hughes AJ, Daniel SE, Kilford L, et al. Accuracy of clinical diagnosis of idiopathic Parkinson's disease: a clinico- pathological study of 100 cases. $\mathcal{F}$ Neurol Neurosurg Psychiatry 1992;55:181-4.

11 Piccini P, Morrish PK, Tujanski N, et al. Dopaminergic function in familial Parkinson's disease. A clinical and ${ }^{18} \mathrm{~F}-$ Dopa positron emission tomography study. Ann Neurol 1997;41:222-9.

12 Vingerhoets FJG, Schulzer M, Calne DB, et al. Which clinical sign of Parkinson's disease best reflects the nigrostriatal lesion? Ann Neurol 1997;41:58-64.

13 Zimmermann R, Deutschl G, Hrning A, et al. Tremors in Parkinson's disease: symptom analysis and rating. Clin Neuropharmacol 1994;17:303-14.

14 Brown P, Corcos DM, Rothwell JC. Does parkinsonian action tremor contribute to muscle weakness in Parkinson's disease? Brain 1997;120:401-8.

15 Henderson JM, Einstein E, Jackson DM, et al. 'Atypical' tremor. Eur Neurol 1995;35:321-6.

16 Lance JW, Schwab RS, Peterson EA. Action tremor and the cogwheel phenomenon in Parkinson's disease. Brain 1963; 86:95-110

17 Teräväinen H, Calne DB. Action tremor in Parkinson's disease. I Neurol Neurosurg Psychiatry 1980;43:257-63.

18 Findley LJ, Gresty MA, Halmagyi GM. Tremor, the cogwheel phenomenon and clonus in Parkinson's disease. $\mathcal{F}$ Neurol Neurosurg Psychiatry 1981;44:534-46.

19 Gresty MA, Findley LJ. Postural and resting tremors in Parkinson's disease. Adv Neurol 1984;40:361-4.

20 Lamarre Y. Animal models of physiological, essential and parkinsonian-like tremors. In: Findley LJ, Capildeo R, eds. Movement disorders: tremor. New York: Oxford University Press, 1984:183-94.

21 Lozano A, Hutchison W, Kiss Z, et al. Methods for microelectrode-guided posteroventral pallidotomy. $\mathcal{F} \mathrm{Neu}$ rosurg 1996;84:194-202.

22 Ondo W, Jankovic J, Lai E, et al. Assessment of motor function following stereotactic pallidotomy. Neurology 1998;50: 266-70.

23 Geraghty JJ, Jankovic J, Zetusky WJ. Association between essential tremor and Parkinson's disease. Ann Neurol 1985; 17:329-33.

24 Lee MS, Kim YD, Im JH, et al. ${ }^{125}$ I-IPT brain SPECT study in essential tremor and Parkinson's disease. Neurology 1999;52:1422-6. 